Article

\title{
Antiproliferative Phenothiazine Hybrids as Novel Apoptosis Inducers against MCF-7 Breast Cancer
}

\author{
Jun-Xia Zhang ${ }^{1,2}$, Jiao-Mei Guo ${ }^{2}$, Ting-Ting Zhang ${ }^{2}$, Hong-Jun Lin ${ }^{2}$, Nai-Song Qi ${ }^{2}$, \\ Zhen-Guo Li ${ }^{2}$, Ji-Chun Zhou ${ }^{2}$ and Zhen-Zhong Zhang ${ }^{1,3, *}$ \\ 1 School of Pharmaceutical science, Zhengzhou University, Zhengzhou 450001, China; zjx312@163.com \\ 2 Department of Pharmacology, Henan Provincial Institute of Food and Drug Control, \\ Zhengzhou 450008, China; ssjsel745288@163.com (J.-M.G.); 18637123006@163.com (T.-T.Z.); \\ 13393723919@163.com (H.-J.L.); 18538293031@163.com (N.-S.Q.); 13298181239@163.com (Z.-G.L.); \\ 18838969948@163.com (J.-C.Z.) \\ 3 Key Laboratory of Targeting Therapy and Diagnosis for Critical Diseases, Henan Province, \\ Zhengzhou 450001, China \\ * Correspondence: zhangzhenzhong@zzu.edu.cn; Tel.: +0371-6338-8226
}

Received: 18 April 2018; Accepted: 17 May 2018; Published: 28 May 2018

\begin{abstract}
We designed a series of novel phenothiazine-1,2,3-triazole hybrids by the molecular hybridization strategy and evaluated their antiproliferative activity against three cancer cell lines (MDA-MB-231, MDA-MB-468 and MCF-7). For the structure-activity relationships, the importance of 1,2,3-triazole and substituents on phenyl ring was explored. Among these phenothiazine-1,2,3-triazole hybrids, compound $9 \mathrm{f}$ showed the most potent inhibitory effect against MCF-7 cells, with an $\mathrm{IC}_{50}$ value of $0.8 \mu \mathrm{M}$. Importantly, compound $9 \mathrm{f}$ could induce apoptosis against MCF-7 cells by regulating apoptosis-related proteins (Bcl-2, Bax, Bad, Parp, and DR5). These potent phenothiazine-1,2,3-triazole hybrids as novel apoptosis inducers might be used as antitumor agents in the future.
\end{abstract}

Keywords: phenothiazine-1,2,3-triazole; MCF-7; apoptosis; DR5

\section{Introduction}

According to the cancer epidemiology data collected in the cancer statistics 2017, breast cancer accounts for about $14.4 \%$ of cancer related death and $29.6 \%$ of new cases in female, and which is the top type of female new cancer cases and the second cause of female cancer related death worldwide after lung cancer [1]. Its poor overall prognosis reflects not only its inherent aggressiveness but also the lack of targeted therapies [2]. Therefore, it is necessary to develop the potent antitumor agents to treat breast cancer.

Phenothiazine is a useful scaffold in antitumor drug design [3-5]. Recently, many phenothiazine derivatives were designed as novel anticancer agents. For example, phenothiazine derivative 1 (Figure 1) proved to be a microtubule-interacting agent with great cell growth inhibition profile $\left(\mathrm{GI}_{50}\right.$ in the nanomolar range on human melanoma-adenocarcinoma MDA-MB-435 cells) [6]. Phenothiazine derivative 2 could arrest tumor cell cycle in the G2/M phase [7]. The 3-hydroxy-4-methoxyphenylethyl derivative 3 was a potent in vitro tubulin polymerization inhibitor but with weak cytotoxicity toward the human colon adenocarcinoma HT29 cell line, in the micromolar range [8]. 


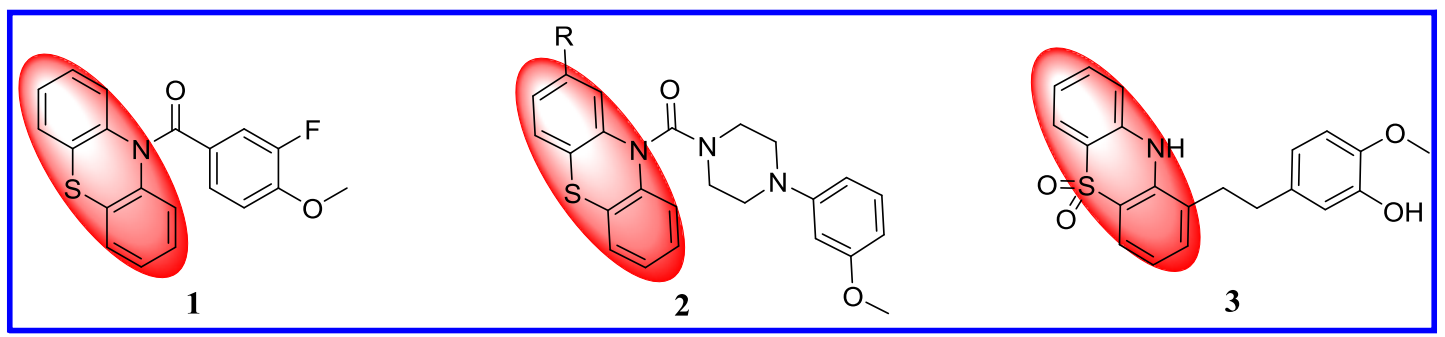

Figure 1. Phenothiazine derivatives as novel anticancer agents.

Triazole derivatives display an ample spectrum of pharmacological activities and are widely employed as pharmaceuticals and agrochemicals [9]. They have been regarded as important skeletons in terms of biological activity and some of them have also shown significant anticancer activity [10]. Benzenesulfonamide 4 (Figure 2) showed an $\mathrm{IC}_{50}$ value of $4.08 \mu \mathrm{M}$ against PC-3 cells and the potent tubulin depolymerization activity in vitro with an $\mathrm{IC}_{50}$ value of $2.41 \mu \mathrm{M}$. Arylamide-1,2,3-triazole 5 exhibited potent antitumor in vitro against MCF-7 cancer cells $\left(\mathrm{IC}_{50}=46 \mathrm{nM}\right)$ [11]. Picolinamide-1,2,3-triazole 6 could induce apoptosis and colony formation at a low micromolar concentration against HT-29 cancer cells [12].

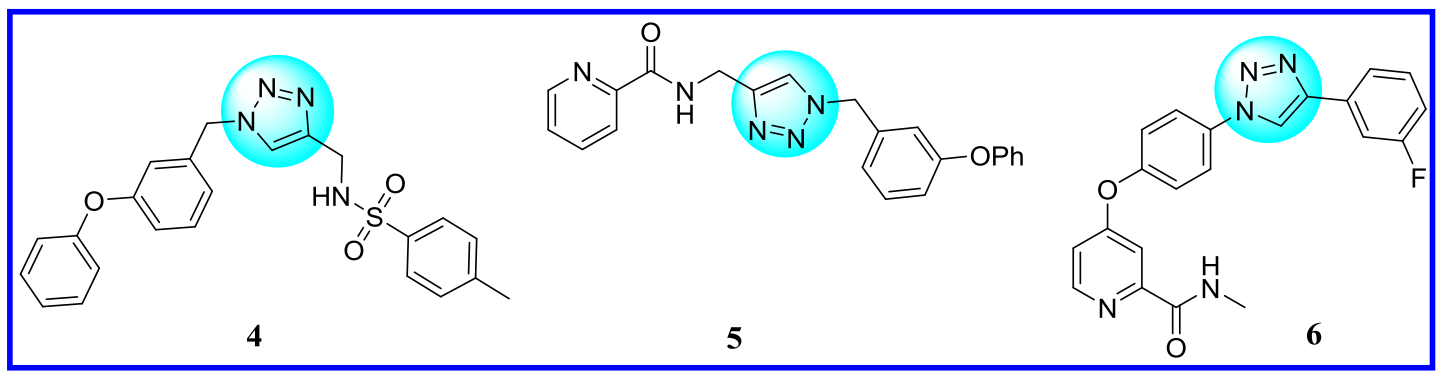

Figure 2. 1,2,3-Triazole derivatives as novel antitumor agents.

Based on the results of 1,2,3-triazole and phenothiazine as antitumor skeletons, we envisioned that a phenothiazine ring linked a 1,2,3-triazole unit would serve as a novel and potent anticancer agents. In this study, the synthesis and antiprolifervative evaluation of phenothiazine-1,2,3-triazole analogues, as well as the inducing apoptosis properties for the most active compound were explored (Figure 3).

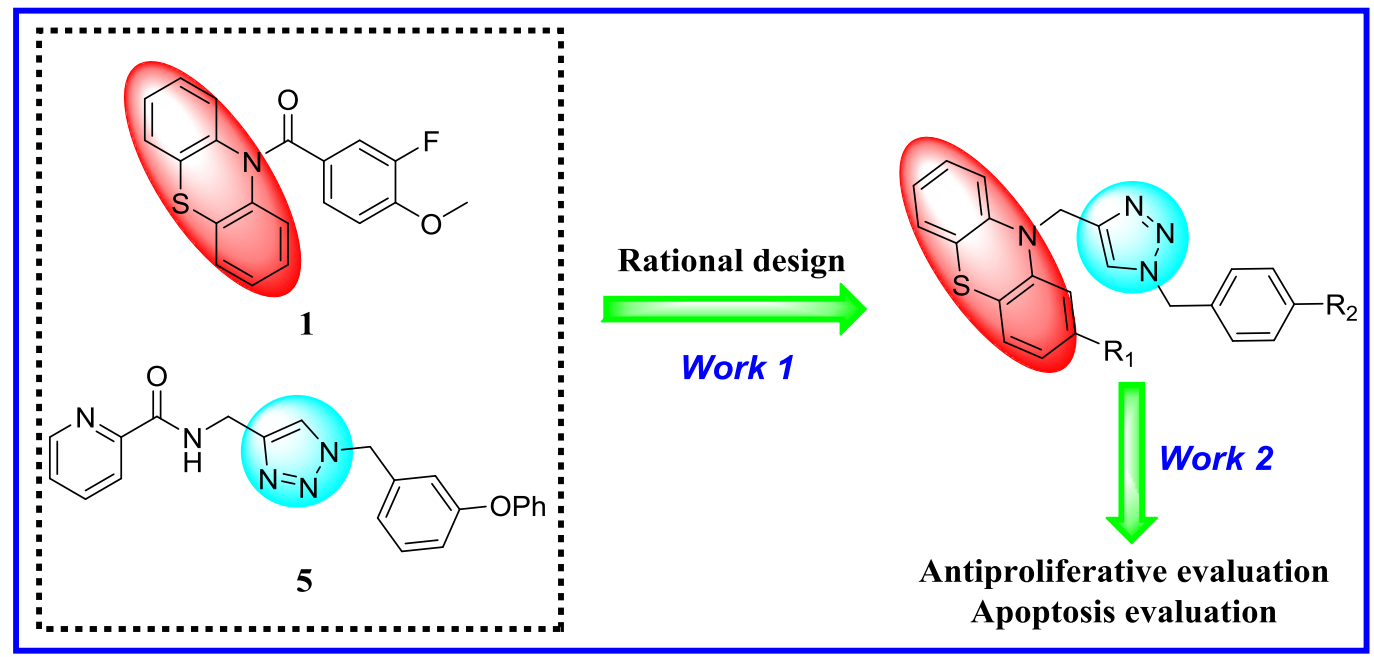

Figure 3. Rational design of target compounds as novel antitumor agents. 


\section{Results and Discussion}

\subsection{Chemistry}

Azide derivatives 7a-7h used in this paper were purchased from Beijing Research Biotechnology Co., Ltd (Beijing, China) (Figure 4). Commercially available phenothiazine was treated with propargyl bromide to provide $\mathbf{8} \mathbf{a}-\mathbf{8 b}$ in the presence of potassium carbonate.

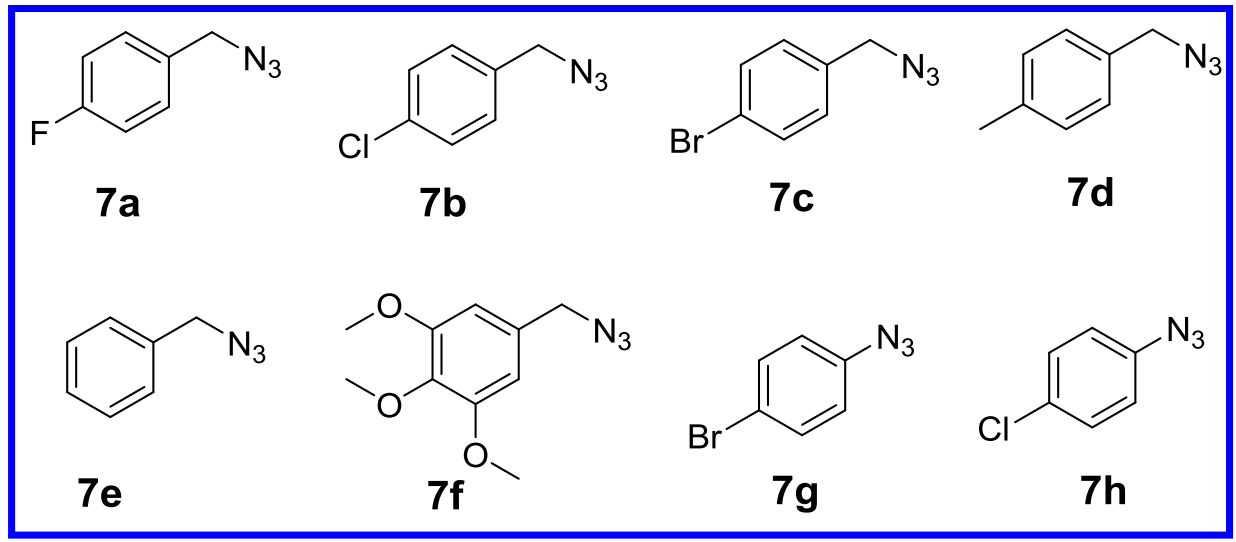

Figure 4. Azide derivatives 7a-7h used in this study.

Azide-alkyne 1,3-dipolar cycloaddition reaction popularly known as the "click chemistry" serves as the highly dependable tool for facile construction of antiproliferative agents [13,14]. These novel phenothiazine hybrids $\mathbf{9 a}-\mathbf{9 k}$ were obtained from alkyne intermediate $\mathbf{8 a}-\mathbf{8 b}$ and azide derivatives $\mathbf{7} \mathbf{a}-\mathbf{7 h}$ using sodium ascorbate and copper sulfate system in $\mathrm{THF} / \mathrm{H}_{2} \mathrm{O}$ (Scheme 1 ). (The spectra of compounds $\mathbf{9 a}-\mathbf{9 k}$ can be seen in the supplementary materials.)

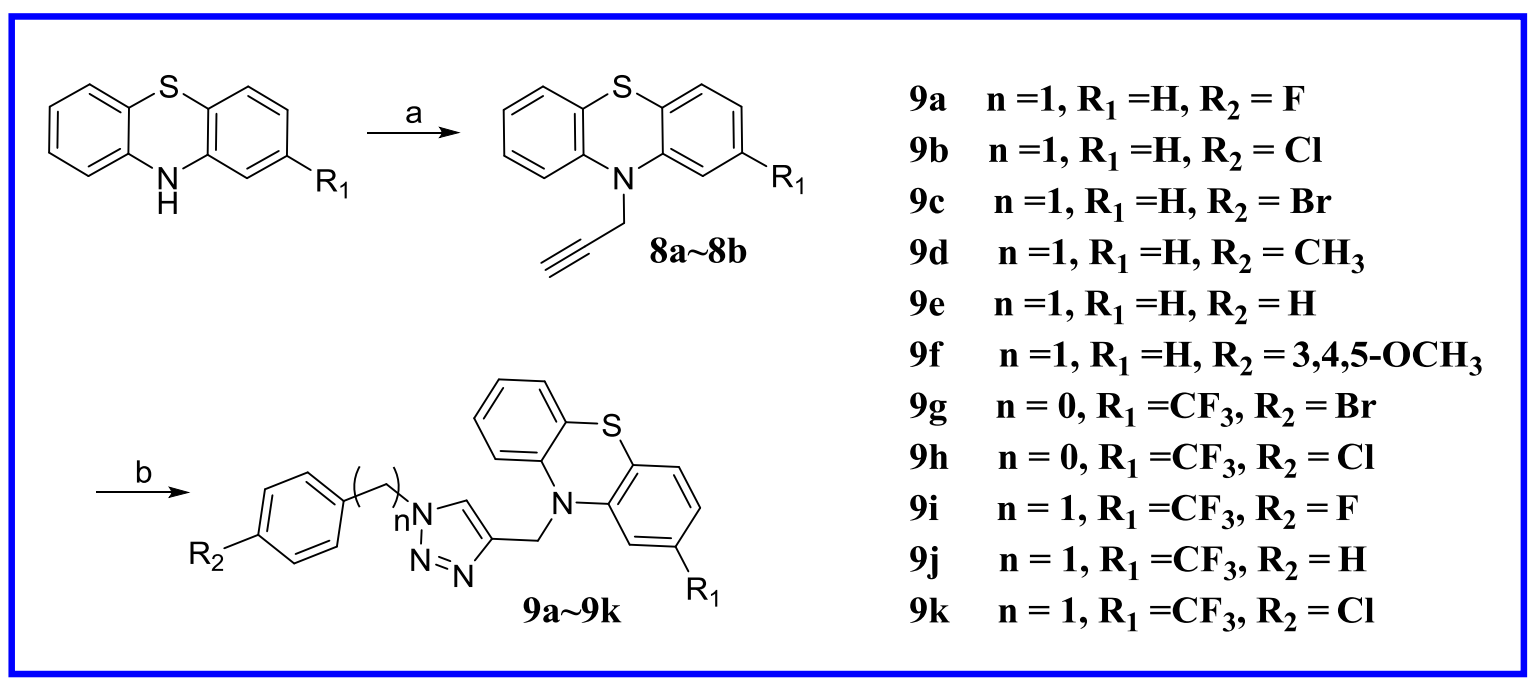

Scheme 1. Synthesis of phenothiazine-1,2,3-triazole hybrids 9a-9k. Reagents and conditions: (a) $\mathrm{K}_{2} \mathrm{CO}_{3}$, dichloromethane, propargyl bromide, reflux; (b) $\mathrm{CuSO}_{4} \cdot 5 \mathrm{H}_{2} \mathrm{O}$, sodium ascorbate, azide derivatives 7a-7h, THF: $\mathrm{H}_{2} \mathrm{O}(1: 1)$, rt.

\subsection{Antiproliferative Activity and SARs}

All the synthesized and target phenothiazine-1,2,3-triazole hybrids 9a-9k were evaluated for their antitumor activity in vitro against three breast cancer cell lines (MDA-MB-468, MDA-MB-231 and MCF-7) using a reported MTT assay [15]. In our antiproliferative experiments, 5-fluorouracil 
(5-Fu) was used as a control $[16,17]$. Table 1 describes the antiproliferative activity results of phenothiazine-1,2,3-triazole hybrids $\mathbf{9 a}-\mathbf{9} \mathbf{k}$ and phenothiazine derivatives $\mathbf{8 a - 8} \mathbf{b}$.

Table 1. Antiproliferative results of phenothiazine-1,2,3-triazole hybrids 9a-9k.

\begin{tabular}{cccc}
\hline \multirow{3}{*}{ Compound } & \multicolumn{3}{c}{$\mathbf{I C}_{\mathbf{5 0}}(\boldsymbol{\mu M})^{\mathbf{a}}$} \\
\cline { 2 - 4 } & MDA-MB-231 & MDA-MB-468 & MCF-7 \\
\hline $\mathbf{8 a}$ & $>100$ & $>100$ & $>100$ \\
$\mathbf{8 b}$ & $>100$ & $>100$ & $>100$ \\
$\mathbf{9 a}$ & $9.0 \pm 0.6$ & $15.7 \pm 0.3$ & $8.6 \pm 1.1$ \\
$\mathbf{9 b}$ & $11.4 \pm 0.2$ & $5.5 \pm 1.7$ & $8.8 \pm 2.0$ \\
$\mathbf{9 c}$ & $14.2 \pm 1.3$ & $9.0 \pm 0.9$ & $11.1 \pm 0.5$ \\
$\mathbf{9 d}$ & $2.0 \pm 0.2$ & $1.6 \pm 0.3$ & $7.3 \pm 1.1$ \\
$\mathbf{9 e}$ & $10.5 \pm 1.0$ & $8.4 \pm 0.8$ & $7.2 \pm 0.2$ \\
$\mathbf{9 f}$ & $1.7 \pm 0.1$ & $1.2 \pm 0.2$ & $0.8 \pm 0.1$ \\
$\mathbf{9 g}$ & $56.3 \pm 0.8$ & $46.4 \pm 2.8$ & $>100$ \\
$\mathbf{9 h}$ & $39.1 \pm 0.6$ & $43.3 \pm 0.7$ & $29.6 \pm 1.3$ \\
$\mathbf{9 i}$ & $11.3 \pm 2.6$ & $11.2 \pm 0.8$ & $14.2 \pm 1.7$ \\
$\mathbf{9 j}$ & $17.4 \pm 1.4$ & $17.6 \pm 2.4$ & $15.3 \pm 0.4$ \\
$\mathbf{9 k}$ & $17.3 \pm 0.9$ & $14.3 \pm 0.8$ & $16.4 \pm 1.1$ \\
$\mathbf{5 - F u}$ & $10.8 \pm 0.3$ & $7.5 \pm 0.2$ & $12.7 \pm 0.6$ \\
\hline
\end{tabular}

a Inhibitory activity was assayed by exposure for $72 \mathrm{~h}$ to substances and expressed as concentration required to inhibit tumor cell proliferation by $50 \%\left(\mathrm{IC}_{50}\right)$. Data are presented as the means \pm SDs of three independent experiments.

To explore the inhibitory effect of 1,2,3-triazole unit, phenothiazines $\mathbf{8 a - 8 b}$ and $\mathbf{9 a}-\mathbf{9} \mathbf{k}$ were evaluated for their antiproliferative activity. When the 1,2,3-triazole group was removed, the inhibitory activity against all the cancer cell lines was decreased $\left(\mathrm{IC}_{50}>100 \mu \mathrm{M}\right)$, indicating the 1,2,3-triazole scaffold is necessary for inhibitory activity. Especially, compound $9 \mathrm{f}$ showed the most potent inhibitory effect against all cell lines with $\mathrm{IC}_{50}$ values ranging from $0.8 \mu \mathrm{M}$ to $1.7 \mu \mathrm{M}$.

In addition, the substituent on the phenothiazine ring may be important for antiproliferative activity. When the hydrogen atom of compounds $\mathbf{9 a - 9}$ f was replaced by a trifluoromethyl group of compounds $9 \mathrm{~g}-\mathbf{9 k}$, the antiproliferative activity was decreased. Compound $\mathbf{9 g}$ displayed the weak antiproliferative activity against MCF-7 cells $(>100 \mu \mathrm{M})$, indicating the significance of the hydrogen atom on the phenothiazine ring for their antiproliferative activity.

The substituent on the phenyl ring also displayed a remarkable effect on the antiproliferative activity against the breast cancer cell lines. Compound $9 \mathrm{c}$ with the 4-bromine atom on the phenyl ring showed more potent antiproliferative activity $(9.0 \mu \mathrm{M})$ than compound $9 \mathrm{a}$ with a 4 -F substituent $(15.7 \mu \mathrm{M})$ against MDA-MB-468 cells, and compound 9 f with a 3,4,5-trimethoxy substituent on the phenyl ring showed more potent antiproliferative activity $(0.8 \mu \mathrm{M})$ than compound $9 \mathbf{d}$ with the $4-\mathrm{CH}_{3}$ substituent $(7.3 \mu \mathrm{M})$ against MCF-7 cell line. When $4-\mathrm{CH}_{3}$ substituent (9d) as an electron-donating group was replaced by the electron-withdrawing groups like $\mathrm{F}, \mathrm{Cl}, \mathrm{Br}(\mathbf{9 a}, \mathbf{9 b}, \mathbf{9 c})$, the antiproliferative activity was decreased against all cancer cell lines. The summary of structure activity relationships is shown in Figure 5. 


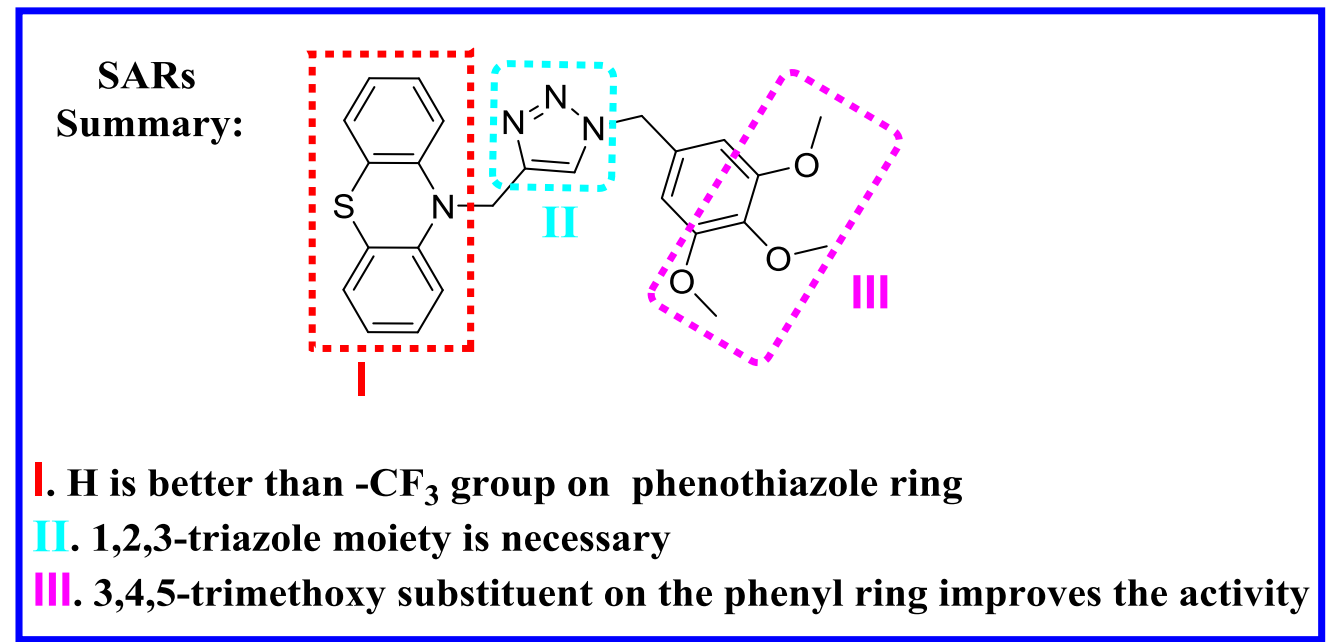

Figure 5. The summary of structure activity relationships.

\subsection{Compound $9 \mathrm{f}$ Induced Morphological Changes of Mcf-7 Cells}

Because of the most potent anticancer activity in vitro against all the selected cancer cell lines among phenothiazine-1,2,3-triazole hybrids, the antiproliferative mechanism of compound $9 \mathrm{f}$ was explored. To explore cytotoxicity of 9f in MCF-7 cells, cell apoptosis was studied by the reported Hoechst 33258 staining assay [18]. After $24 \mathrm{~h}$ incubation with 9 f at $0.5 \mu \mathrm{M}$ and $1 \mu \mathrm{M}$, characteristic apoptotic morphological changes could be served by fluorescence microscope, including cell rounding, chromain shrinkage and formation of apoptotic bodies (Figure 6). Therefore, target compound $9 \mathrm{f}$ could cause morphological changes of MCF-7 cancer cells possibly by inducing apoptosis process.

\section{Control}

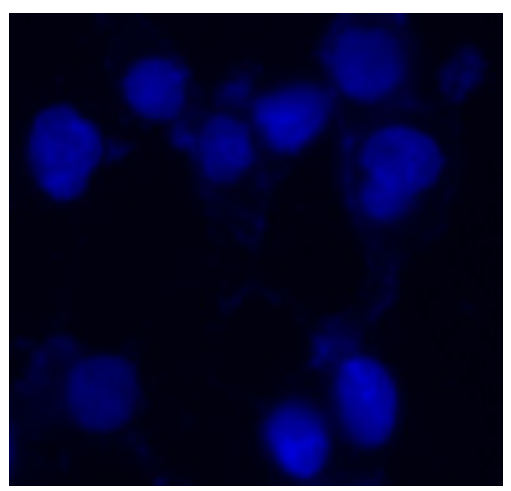

$0.5 \mu \mathrm{M}$

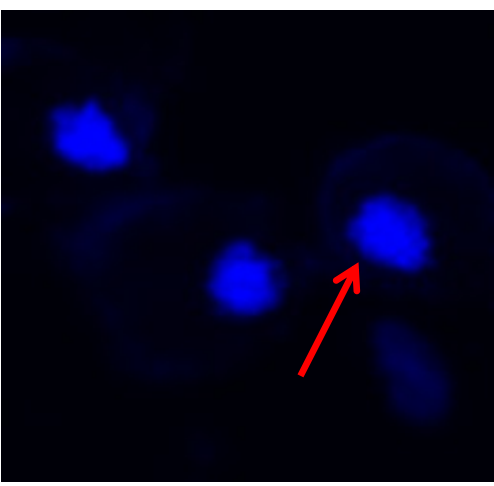

$1 \mu \mathrm{M}$

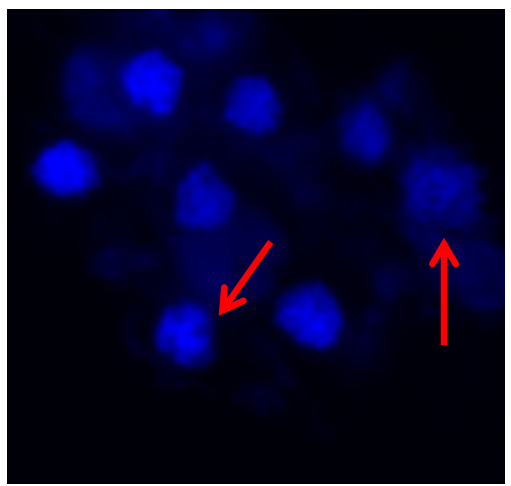

Figure 6. MCF-7 cells treated by $9 \mathrm{f}$ showed typical apoptotic morphologies.

\subsection{Compound 9 Induced Apoptosis of Mcf-7 Cells}

Based on the biological experiments above, the apoptosis property of compound 9f was investigated against MCF-7 cells (Figure 7). After treatment with different concentrations of compound 9f $(0,0.5$ and $1 \mu \mathrm{M})$ for $24 \mathrm{~h}$, MCF-7 cells were stained with propidium Iodide and Annexin V Binding Buffer, and then analyzed by the flow cytometry. As illustrated in Figure 7, the percentage of apoptotic cells by treatment of $1 \mu \mathrm{M}$ compound $9 \mathrm{f}$ was changed obviously from the control group. 

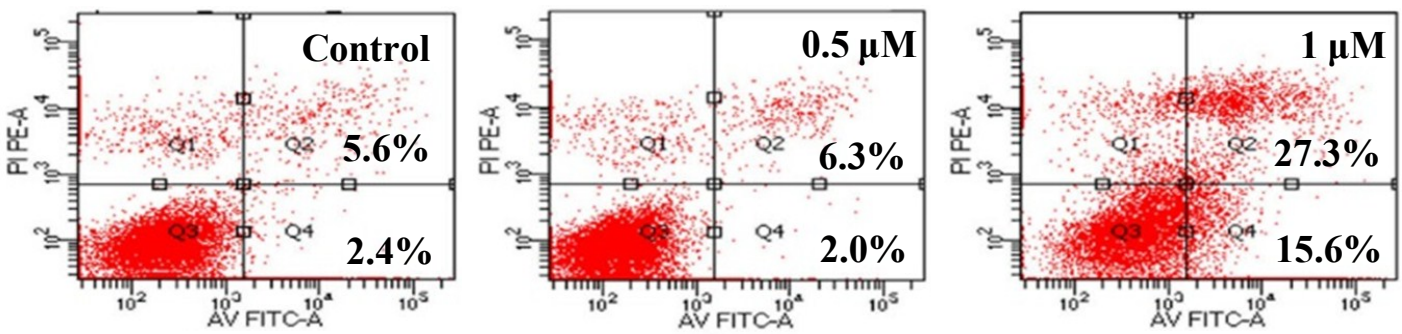

\begin{tabular}{|c|c|c|c|}
\hline 9f $(\mu \mathrm{M})$ & 0 & 0.5 & 1 \\
\hline Early apoptosis $(\%)$ & 2.4 & 2.0 & 15.6 \\
\hline Late apoptosis $(\%)$ & 5.6 & 6.3 & 27.3 \\
\hline
\end{tabular}

Figure 7. Compound 9f could induce apoptosis of MCF-7 cells.

\subsection{Compound 9f Related Apoptosis-Related Proteins}

The changes of morphological features, such as cell shrinkage, chromatin condensation, nuclear membrane blebbing are the characteristics of apoptotic cells [19]. Based on the above results, we explored the effect of compound 9 f on celluar apoptosis. Bcl-2 family proteins could dictate death decisions or cellular survival by regulating the integrity of the mitochondrial outer membrane [20]. Recent years, Bcl-2 family proteins have emerged as anticancer targets [21].

Due to the potent cytotoxic activity against MCF-7 cell line, compound 9f was evaluated the related-apoptosis proteins. In western blot assay, compound $9 \mathrm{f}$ increased the level of Bad, Bax and DR5 and reduced the level of Bcl-2 and Parp which could induce apoptosis of MCF-7 cells (Figure 8).

\section{Control $0.5 \mu \mathrm{M} \quad 1 \mu \mathrm{M}$}

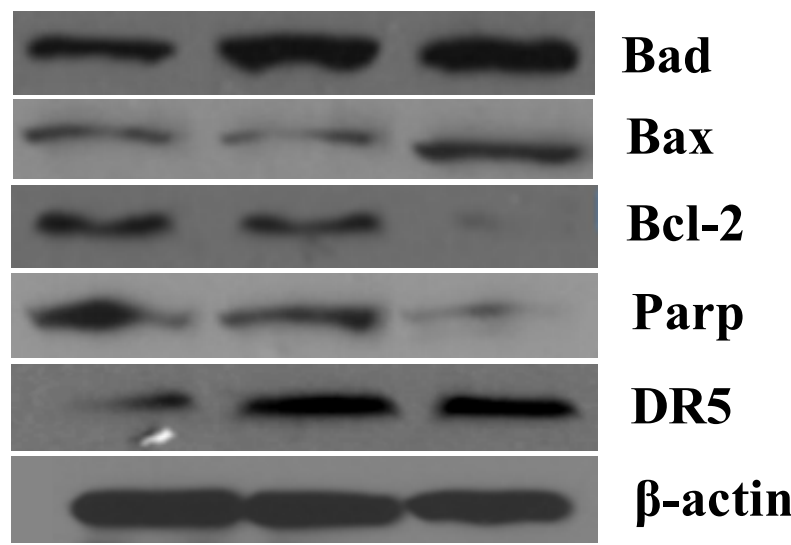

Figure 8. Expression of apoptosis-related proteins in MCF-7 cells after treatment by compound 9f for $24 \mathrm{~h}$.

\section{Experimental Section}

\subsection{Chemistry}

Reagents and solvents used were purchased from Beijing Research Biotechnology Co., Ltd. (Beijing, China). All nMR spectra were obtained from a Bruker DPX $400 \mathrm{MHz}$ spectrometer (Agilent, Santa Clara, CA, USA) (internal standard was TMS). Mass spectra (MS) were recorded on Esquire 3000 mass spectrometer by electrospray ionization (ESI) (Varian, Palo Alto, CA, USA) for all phenothiazole-1,2,3-triazole hybrids. 


\subsubsection{Phenothiazine Derivatives $\mathbf{8 a}-\mathbf{8 b}$}

To a stirred solution of phenothiazine or 2-(trifluoromethyl)-10H-phenothiazine (3 mmol) in dichloromethane $(20 \mathrm{~mL}), \mathrm{K}_{2} \mathrm{CO}_{3}(5 \mathrm{mmol})$ and propargyl bromide $(5 \mathrm{mmol})$ were added carefully and the reaction mixture was refluxed for $7 \mathrm{~h}$. After the reaction, the system was concentrated under vacuum, the residue was dissolved in EtOAc $(40 \mathrm{~mL})$ and washed by pure water and brine, and dried over anhydrous $\mathrm{Na}_{2} \mathrm{SO}_{4}$ and concentrated under vacuum to afford crude products $\mathbf{8 a}-\mathbf{8 b}$, which were used in the next reaction without further purification.

\subsubsection{General Method to Synthesize Phenothiazine-1,2,3-Triazole Hybrids 9a-9k}

Alkyne intermediates $\mathbf{8 a - 8 b}(3 \mathrm{mmol})$, azide derivatives $\mathbf{7 a}-\mathbf{7 h}(6 \mathrm{mmol}), \mathrm{CuSO}_{4} .5 \mathrm{H}_{2} \mathrm{O}(1.2 \mathrm{mmol})$ and sodium ascorbate $(0.6 \mathrm{mmol})$ were dissolved in $\mathrm{THF} / \mathrm{H}_{2} \mathrm{O}(20 \mathrm{~mL} / 20 \mathrm{~mL})$. The system was reacted for $8 \mathrm{~h}$ at room temperature. After the reaction, the product was filtered to afford the products $9 \mathbf{a}-9 \mathbf{k}$, which were purified with column chromatography on silica gel (hexane/EtOAc $=9 / 1$ ).

10-\{[1-(4-Fluorobenzyl)-1H-1,2,3-triazol-4-yl]methyl\}-10H-phenothiazine (9a). Yield: 87\%. White solid. Mp: $166-167^{\circ} \mathrm{C} .{ }^{1} \mathrm{H}$ nMR (400 MHz, DMSO-d 6 ) $\delta 8.01$ (s, 1H, CHN), 7.29-7.21 (m, 2H, Ar), 7.19-7.06 (m, 6H, Ar), 6.99-6.85 (m, 4H, Ar), 5.54 (s, 2H, $\left.\mathrm{PhCH}_{2}\right), 5.14$ (s, 2H, $\left.\mathrm{NCH}_{2}\right) .{ }^{13} \mathrm{C} \mathrm{nMR}(100 \mathrm{MHz}$, DMSO-d $\left._{6}\right) \delta 162.96,160.53,144.02,143.77,132.42,132.39,129.87,129.78,127.42,126.74,123.64,122.64$, 122.62, 115.71, 115.55, 115.34, 51.86, 43.86. HR-MS (ESI): Calcd. $\mathrm{C}_{22} \mathrm{H}_{18} \mathrm{FN}_{4} \mathrm{~S},[\mathrm{M}+\mathrm{H}]^{+} \mathrm{m} / z: 389.1236$, found: 389.1239 .

10-\{[1-(4-Chlorobenzyl)-1H-1,2,3-triazol-4-yl]methyl\}-10H-phenothiazine (9b). Yield: 69\%. White solid. Mp: $150-151^{\circ} \mathrm{C} .{ }^{1} \mathrm{H}$ nMR (400 MHz, DMSO-d 6 ) $\delta 8.02$ (s, 1H, CHN), 7.57-7.46 (m, 2H, Ar), 7.19-7.04 $(\mathrm{m}, 6 \mathrm{H}, \mathrm{Ar}), 6.92(\mathrm{t}, J=7.3 \mathrm{~Hz}, 4 \mathrm{H}, \mathrm{Ar}), 5.54\left(\mathrm{~s}, 2 \mathrm{H}, \mathrm{PhCH}_{2}\right), 5.15\left(\mathrm{~s}, 2 \mathrm{H}, \mathrm{NCH}_{2}\right) .{ }^{13} \mathrm{C} \mathrm{nMR}(100 \mathrm{MHz}$, DMSO-d $\left._{6}\right) \delta 144.01,143.80,135.60,131.53,129.75,127.43,126.75,123.79,122.65,122.63,121.19,115.71$, 51.91, 43.87. HR-MS (ESI): Calcd. $\mathrm{C}_{22} \mathrm{H}_{18} \mathrm{ClN}_{4} \mathrm{~S},[\mathrm{M}+\mathrm{H}]^{+} \mathrm{m} / z: 405.0941$, found: 405.0948 .

10-\{[1-(4-Bromobenzyl)-1H-1,2,3-triazol-4-yl]methyl\}-10H-phenothiazine (9c). Yield: 77\%. White solid. Mp: 154-155 ${ }^{\circ} \mathrm{C} .{ }^{1} \mathrm{H}$ nMR $\left(400 \mathrm{MHz}, \mathrm{DMSO}-\mathrm{d}_{6}\right) \delta 8.02$ (s, 1H, CHN), 7.49-7.28 (m, 2H, Ar), 7.19 (d, J = 8.5 Hz, 2H, Ar), 7.16-7.05 (m, 4H, Ar), 6.93 (t, J = 7.2 Hz, 4H, Ar), $5.56\left(\mathrm{~s}, 2 \mathrm{H}, \mathrm{PhCH}_{2}\right), 5.15\left(\mathrm{~s}, 2 \mathrm{H}, \mathrm{NCH}_{2}\right)$. ${ }^{13} \mathrm{C}$ nMR $\left(100 \mathrm{MHz}, \mathrm{DMSO}-\mathrm{d}_{6}\right) \delta 144.01,143.81,135.18,132.66,129.44,128.61,127.43,126.75,123.78$, $122.65,122.63,115.71,51.85,43.86$. HR-MS (ESI): Calcd. $\mathrm{C}_{22} \mathrm{H}_{18} \mathrm{BrN}_{4} \mathrm{~S},[\mathrm{M}+\mathrm{H}]^{+} \mathrm{m} / z: 449.0436$, found: 449.0440 .

10-\{[1-(4-Methylbenzyl)-1H-1,2,3-triazol-4-yl]methyl\}-10H-phenothiazine (9d). Yield: 90\%. White solid. Mp: $151-152{ }^{\circ} \mathrm{C} .{ }^{1} \mathrm{H}$ nMR (400 MHz, DMSO-d 6 ) $\delta 7.98$ (s, 1H, CHN), 7.23-7.00 (m, 8H, Ar), 7.00-6.79 $(\mathrm{m}, 4 \mathrm{H}, \mathrm{Ar}), 5.49$ (s, 2H, $\left.\mathrm{PhCH}_{2}\right), 5.13\left(\mathrm{~s}, 2 \mathrm{H}, \mathrm{NCH}_{2}\right), 2.26\left(\mathrm{~s}, 3 \mathrm{H}, \mathrm{CH}_{3}\right) .{ }^{13} \mathrm{C} \mathrm{nMR}\left(100 \mathrm{MHz}, \mathrm{DMSO}_{6}\right)$ $\delta 144.03,143.65,137.25,133.15,129.14,127.53,127.41,126.73,123.57,122.61,115.70,52.45,43.86,20.63$. HR-MS (ESI): Calcd. $\mathrm{C}_{23} \mathrm{H}_{21} \mathrm{~N}_{4} \mathrm{~S},[\mathrm{M}+\mathrm{H}]^{+} \mathrm{m} / z$ : 385.1487, found: 385.1489 .

10-\{[1-Benzyl-1H-1,2,3-triazol-4-yl]methyl\}-10H-phenothiazine (9e). Yield: 82\%. White solid. Mp: 150-152 ${ }^{\circ} \mathrm{C}$. ${ }^{1} \mathrm{H}$ nMR (400 MHz, DMSO-d $\left.{ }_{6}\right) \delta 8.01$ (s, 1H, CHN), 7.37-7.23 (m, 3H, Ar), 7.20-7.05 (m, 6H, Ar),

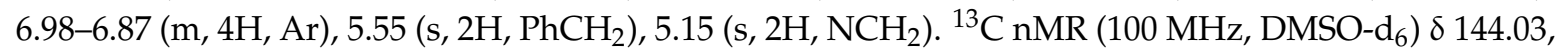
143.73, 136.18, 128.61, 127.92, 127.45, 127.41, 126.74, 123.75, 122.65, 122.62, 115.72, 52.63, 43.87. HR-MS (ESI): Calcd. $\mathrm{C}_{22} \mathrm{H}_{19} \mathrm{~N}_{4} \mathrm{~S},[\mathrm{M}+\mathrm{H}]^{+} \mathrm{m} / z$ : 371.1330, found: 371.1339 .

10-\{[1-(3,4,5-Trimethoxybenzyl)-1H-1,2,3-triazol-4-yl]methyl\}-10H-phenothiazine (9f). Yield: 88\%. White solid. Mp: $146-147{ }^{\circ} \mathrm{C} .{ }^{1} \mathrm{H}$ nMR $\left(400 \mathrm{MHz}, \mathrm{DMSO}-\mathrm{d}_{6}\right) \delta 8.05$ (s, 1H, CHN), 7.19-7.01 (m, 4H, Ar), 7.01-6.83 (m, 4H, Ar), 6.55 (s, 2H, Ar), 5.46 (s, 2H, $\left.\mathrm{PhCH}_{2}\right), 5.14$ (s, 2H, NCH 2$), 3.67$ (s, 6H, OCH $\mathrm{OCH}_{3}, 3.62$ $\left(\mathrm{s}, 3 \mathrm{H}, \mathrm{OCH}_{3}\right) .{ }^{13} \mathrm{C}$ nMR $\left(100 \mathrm{MHz}, \mathrm{DMSO}-\mathrm{d}_{6}\right) \delta 152.92,144.03,143.66,137.12,131.56,127.39,126.75$, 123.58, 122.62, 122.52, 115.64, 105.12, 59.94, 55.80, 52.87, 43.80. HR-MS (ESI): Calcd. $\mathrm{C}_{25} \mathrm{H}_{25} \mathrm{~N}_{4} \mathrm{O}_{3} \mathrm{~S}$, $[\mathrm{M}+\mathrm{Na}]^{+} \mathrm{m} / z: 483.1467$, found: 483.1468 . 
10-\{[1-(4-Bromophenyl)-1H-1,2,3-triazol-4-yl]methyl\}-2-\{trifluoromethyl\}-10H-phenothiazine (9g). Yield: 93\%. White solid. Mp: $161-162{ }^{\circ} \mathrm{C} .{ }^{1} \mathrm{H}$ nMR $\left(400 \mathrm{MHz}, \mathrm{DMSO}-\mathrm{d}_{6}\right) \delta 8.84(\mathrm{~s}, 1 \mathrm{H}, \mathrm{CHN}), 7.86(\mathrm{~d}, J=8.9 \mathrm{~Hz}, 2 \mathrm{H}$, Ar), $7.78(\mathrm{~d}, J=8.9 \mathrm{~Hz}, 2 \mathrm{H}, \mathrm{Ar}), 7.35(\mathrm{~d}, J=8.0 \mathrm{~Hz}, 1 \mathrm{H}, \mathrm{Ar}), 7.26(\mathrm{~d}, J=7.5 \mathrm{~Hz}, 2 \mathrm{H}, \mathrm{Ar}), 7.21-7.12(\mathrm{~m}, 2 \mathrm{H}$, Ar), $7.04(\mathrm{~d}, J=8.0 \mathrm{~Hz}, 1 \mathrm{H}, \mathrm{Ar}), 6.98(\mathrm{t}, J=7.3 \mathrm{~Hz}, 1 \mathrm{H}, \mathrm{Ar}), 5.30\left(\mathrm{~s}, 2 \mathrm{H}, \mathrm{NCH}_{2}\right) .{ }^{13} \mathrm{C} \mathrm{nMR}(100 \mathrm{MHz}$, DMSO-d 6 ) $\delta 144.58,144.35,143.29,135.63,132.76,128.40,128.22,127.97,127.32,126.92,125.41,123.36$, 122.70, $121.73(\mathrm{~d}, J=14.8 \mathrm{~Hz}), 121.29,119.21,116.16,112.03,43.69$. HR-MS (ESI): Calcd. $\mathrm{C}_{22} \mathrm{H}_{15} \mathrm{BrF}_{3} \mathrm{~N}_{4} \mathrm{~S}$, $[\mathrm{M}+\mathrm{H}]^{+} \mathrm{m} / z: 503.0153$, found: 503.0158 .

10-\{[1-(4-Chlorophenyl)-1H-1,2,3-triazol-4-yl]methyl\}-2-\{trifluoromethyl\}-10H-phenothiazine (9h). Yield: 90\%. White solid. Mp: $153-155{ }^{\circ} \mathrm{C} .{ }^{1} \mathrm{H}$ nMR (400 MHz, DMSO-d 6 ) $\delta 8.84$ (s, 1H, CHN), 7.99-7.86 (m, 2H, Ar), 7.73-7.56 (m, 2H, Ar), 7.35 (d, J = 7.7 Hz, 1H, Ar), 7.26 (d, J = 8.4 Hz, 2H, Ar), 7.21-7.12 (m, 2H, Ar), 7.04 $(\mathrm{d}, J=7.9 \mathrm{~Hz}, 1 \mathrm{H}, \mathrm{Ar}), 6.99(\mathrm{t}, J=7.4 \mathrm{~Hz}, 1 \mathrm{H}, \mathrm{Ar}), 5.31\left(\mathrm{~s}, 2 \mathrm{H}, \mathrm{NCH}_{2}\right) .{ }^{13} \mathrm{C} \mathrm{nMR}\left(100 \mathrm{MHz}, \mathrm{DMSO}-\mathrm{d}_{6}\right)$ $\delta 144.58,144.33,143.30,135.23,132.93,129.83,128.41,128.22,127.97,127.91,127.31,126.92,123.36$, $121.83,121.66,121.56,119.20,116.16,112.03,43.58$. HR-MS (ESI): Calcd. $\mathrm{C}_{22} \mathrm{H}_{15} \mathrm{ClF}_{3} \mathrm{~N}_{4} \mathrm{~S},[\mathrm{M}+\mathrm{H}]^{+} \mathrm{m} / z$ : 459.0658, found: 459.0662 .

10-\{[1-(4-Fluorobenzyl)-1H-1,2,3-triazol-4-yl]methyl\}-2-\{trifluoromethyl\}-10H-phenothiazine (9i). Yield: 81\%. White solid. Mp: $134-136{ }^{\circ} \mathrm{C} .{ }^{1} \mathrm{H}$ nMR $\left(400 \mathrm{MHz}, \mathrm{DMSO}_{6}\right) \delta 8.07$ (s, 1H, CHN), 7.33 (d, J = 7.9 Hz,

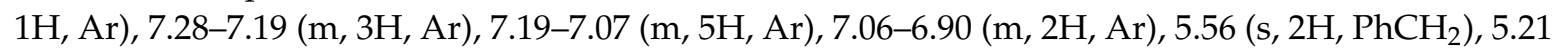
$\left(\mathrm{s}, 2 \mathrm{H}, \mathrm{NCH}_{2}\right) .{ }^{13} \mathrm{C}$ nMR $\left(100 \mathrm{MHz}, \mathrm{DMSO}-\mathrm{d}_{6}\right) \delta 144.44,143.37,143.27,132.41,132.38,129.75,129.66$, 127.92, 127.30, 126.90, 125.35, 123.68, 123.31, 121.53, 119.16, 116.11, 115.49, 115.28, 111.93, 51.89, 43.88. HR-MS (ESI): Calcd. $\mathrm{C}_{23} \mathrm{H}_{17} \mathrm{~F}_{4} \mathrm{~N}_{4} \mathrm{~S},[\mathrm{M}+\mathrm{H}]^{+} \mathrm{m} / z$ : 457.1110 , found: 457.1118 .

10-\{[1-Benzyl-1H-1,2,3-triazol-4-yl]methyl\}-2-\{trifluoromethyl\}-10H-phenothiazine (9j). Yield: 79\%. White solid. Mp: ${ }^{138-139}{ }^{\circ} \mathrm{C} .{ }^{1} \mathrm{H}$ nMR (400 MHz, DMSO-d 6 ) $\delta 8.07$ (s, 1H, CHN), 7.39-7.27 (m, 4H, Ar), 7.24 $(\mathrm{d}, J=8.1 \mathrm{~Hz}, 1 \mathrm{H}, \mathrm{Ar}), 7.20-7.07$ (m, 5H, Ar), 7.03-6.93 (m, 2H, Ar), 5.58 (s, 2H, PhCH $), 5.22$ (s, $2 \mathrm{H}$, $\left.\mathrm{NCH}_{2}\right) .{ }^{13} \mathrm{C}$ nMR $\left(100 \mathrm{MHz}, \mathrm{DMSO}-\mathrm{d}_{6}\right) \delta 144.45,143.38,143.21,136.19,128.56,128.24,128.18,127.90$, 127.30, 127.24, 126.89, 125.37, 123.82, 123.31, 122.66, 121.51, 119.08, 116.12, 111.97, 52.62, 43.92. HR-MS (ESI): Calcd. $\mathrm{C}_{23} \mathrm{H}_{18} \mathrm{~F}_{3} \mathrm{~N}_{4} \mathrm{~S},[\mathrm{M}+\mathrm{H}]^{+} \mathrm{m} / z$ : 439.1204, found: 439.1208 .

10-\{[1-(4-Chlorobenzyl)-1H-1,2,3-triazol-4-yl]methyl\}-2-\{trifluoromethyl\}-10H-phenothiazine (9k). Yield: 86\%. White solid. Mp: $140-142{ }^{\circ} \mathrm{C} .{ }^{1} \mathrm{H}$ nMR $\left(400 \mathrm{MHz}, \mathrm{DMSO}-\mathrm{d}_{6}\right) \delta 8.08$ (s, 1H, CHN), 7.43-7.28 (m, 3H, Ar), $7.24(\mathrm{~d}, J=8.0 \mathrm{~Hz}, 1 \mathrm{H}, \mathrm{Ar}), 7.17$ (ddd, $J=5.7,3.8,2.4 \mathrm{~Hz}, 4 \mathrm{H}, \mathrm{Ar}), 7.12$ (s, 1H, Ar), 7.05-6.85 (m, 2H, Ar), $5.58\left(\mathrm{~s}, 2 \mathrm{H}, \mathrm{PhCH}_{2}\right), 5.22\left(\mathrm{~s}, 2 \mathrm{H}, \mathrm{NCH}_{2}\right) .{ }^{13} \mathrm{C}$ nMR $\left(100 \mathrm{MHz}, \mathrm{DMSO}-\mathrm{d}_{6}\right) \delta 144.44,143.31,135.17,132.66$, $129.31,128.54,128.27,128.17,127.92,127.85,127.31,126.90,123.82,123.31,121.55,119.13,119.09 .116 .12$, 111.89, 51.88, 43.89. HR-MS (ESI): Calcd. $\mathrm{C}_{23} \mathrm{H}_{17} \mathrm{ClF}_{3} \mathrm{~N}_{4} \mathrm{~S},[\mathrm{M}+\mathrm{H}]^{+} \mathrm{m} / z: 473.0815$, found: 473.0819 . This compound was also reported in the previous paper [22].

\subsection{Biology}

\subsubsection{Antiproliferative Activity}

The all breast cancer cell lines were maintained in the mixture of 89\% RPMI1640 medium, 10\% fetal bovine serum and $1 \%$ penicillinestreptomycin. All cell lines were purchased from the China Center for Type Culture Collection (Shanghai, China). The cancer cells were seeded into 96-well plates (5000 cells per well). After $24 \mathrm{~h}$ incubation, the culture medium was removed and replaced with medium containing the candidate compounds. The cells were incubated for another $72 \mathrm{~h}$. Then, $30 \mu \mathrm{L}$ (3-(4,5-dimethylthiazol-2-yl)-2,5-diphenyltetrazo lium bromide) solution $(5 \mathrm{mg} / \mathrm{mL})$ was added and incubated for $2 \mathrm{~h}$. Dimethyl sulfoxide $(40 \mu \mathrm{L})$ was added to each well to dissolve the dark blue formazan; the absorbance was measured using a microplate reader at awavelength of $570 \mathrm{~nm}$. 


\subsubsection{Apoptosis Analysis with Hoechst 33258 Staining}

Near-confluent MCF-7 cells were seeded into a 6-well plate $\left(5.0 \times 10^{6}\right.$ cells/well). After incubation for $24 \mathrm{~h}$ with the test compound, Hoechst 33258 was added to the culture medium to give final concentrations of $5 \mathrm{mg} / \mathrm{mL}$, respectively. The cells were incubated with the staining mixture for $30 \mathrm{~min}$ at $37^{\circ} \mathrm{C}$ and then the cells were observed under a fluorescence microscope (Leica Microsystems AG, Wetzlar, Germany). Apoptotic cells were examined and identified according to the condensation and fragmentation of their nuclei by fluorescence microscopy. Hoechst 33258 permeates all the cells and makes the nuclei appear blue. Apoptosis was revealed by nuclear changes such as chromatin condensation and nuclear fragmentation.

\subsubsection{Apoptosis Analysis with Western Blot}

MCF-7 cells were cultured with different concentrations of compound 9f for $24 \mathrm{~h}$, both adherent and floating cells were collected, and then Western blot analysis was performed. Briefly, Cells were lysed in cell lysis buffer [1\% NP-40, 0.1\% sodium dodecyl sulfate (SDS), $150 \mathrm{mM} \mathrm{NaCl}, 25 \mathrm{mM}$ TriseHCl, $1 \%$ deoxycholic acid sodium salt, $1 \%$ PMSF] for $30 \mathrm{~min}$ on ice. Total protein contents were measured by protein assay reagent. The membrane was indicated primary antibodies at $4{ }^{\circ} \mathrm{C}$. After that, the membrane was incubated with secondary antibodies, goat anti-rabbit and goat anti-mouse IgG conjugated.

\section{Conclusions}

We designed and synthesized a series of novel phenothiazine-1,2,3-triazole hybrids and evaluated their anticancer activity in vitro against three selected cancer cell lines (MDA-MB-468, MDA-MB-231 and MCF-7). Among these hybrids, compound $9 f$ showed the most excellent anticancer activity in vitro with an $\mathrm{IC}_{50}$ value of $0.8 \mu \mathrm{M}$ against MCF-7 cancer cells. Biological mechanism displayed that $9 \mathrm{f}$ induced morphological change and apoptosis against MCF-7 cells by increasing the level of Bad, Bax and DR5 and decreasing the level of Bcl-2 and Parp.

Supplementary Materials: The spectra of compounds $9 \mathbf{a}-\mathbf{9 k}$ are available online.

Author Contributions: J.-X.Z., J.-M.G., T.-T.Z., H.-J.L., N.-S.Q., and Z.-G.L. performed the synthetic work, J.-X.Z., J.-C.Z., and Z.-Z.Z. were responsible for the direction of the biological research. All authors read and approved the final manuscript.

Funding: This research was funded by the scientific and technological project of Henan Province: 162102310067.

Acknowledgments: This work was supported by the scientific and technological project of Henan Province (No. 162102310067).

Conflicts of Interest: The authors declare no conflict of interest.

\section{References}

1. Siegel, R.L.; Miller, K.D.; Jemal, A. Cancer statistics. CA-Cancer J. Clin. 2017, 67, 7-30. [CrossRef] [PubMed]

2. Papa, A.; Caruso, D.; Tomao, S.; Rossi, L.; Zaccarelli, E.; Tomao, F. Triple-negative breast cancer: Investigating potential molecular therapeutic target. Expert Opin. Ther. Targets 2015, 19, 55-75. [CrossRef] [PubMed]

3. Wu, C.H.; Bai, L.Y.; Tsai, M.H.; Chu, P.C.; Chiu, C.F.; Chen, M.Y.; Chiu, S.J.; Chiang, J.H.; Weng, J.R. Pharmacological exploitation of the phenothiazine antipsychotics to develop novel antitumor agents—A drug repurposing strategy. Sci. Rep. 2016, 6, 27540. [CrossRef] [PubMed]

4. Jeleń, M.; Pluta, K.; Zimecki, M.; Morak-Młodawska, B.; Artym, J.; Kocięba, M. 6-Substituted 9-fluoroquino [3,2-b]benzo[1,4]thiazines display strong antiproliferative and antitumor properties. Eur. J. Med. Chem. 2015, 89, 411-420. [CrossRef] [PubMed]

5. Maddila, S.; Naicker, K.; Momin, M.I.K.; Rana, S.; Gorle, S.; Maddila, S.; Yalagala, K.; Singh, M.; Koorbanally, N.A.; Jonnalagadda, S.B. Novel 2-(1-(substitutedbenzyl)-1H-tetrazol-5-yl)-3-phenylacrylonitrile derivatives: Synthesis, in vitro antitumor activity and computational studies. Med. Chem. Res. 2016, 25, 283-291. [CrossRef] 
6. Ghinet, A.; Moise, I.-M.; Rigo, B.; Homerin, G.; Farce, A.; Dubois, J.; Bîcu, E. Studies on phenothiazines: New microtubule-interacting compounds with phenothiazine A-ring as potent antineoplastic agents. Bioorg. Med. Chem. 2016, 24, 2307-2317. [CrossRef] [PubMed]

7. Prinz, H.; Ridder, A.K.; Vogel, K.; Böhm, K.J.; Ivanov, I.; Ghasemi, J.B.; Aghaee, E.; Müller, K. N-Heterocyclic (4-Phenylpiperazin-1-yl)methanones Derived from Phenoxazine and Phenothiazine as Highly Potent Inhibitors of Tubulin Polymerization. J. Med. Chem. 2017, 60, 749-766. [CrossRef] [PubMed]

8. Verones, V.; Flouquet, N.; Lecoeur, M.; Lemoine, A.; Farce, A.; Baldeyrou, B.; Mahieu, C.; Wattez, N.; Lansiaux, A.; Goossens, J.-F.; et al. Synthesis, antiproliferative activity and tubulin targeting effect of acridinone and dioxophenothiazine derivatives. Eur. J. Med. Chem. 2013, 59, 39-47. [CrossRef] [PubMed]

9. Kamal, A.; Shankaraiah, N.; Devaiah, V.; Laxma Reddy, K.; Juvekar, A.; Sen, S.; Kurian, N.; Zingde, S. Synthesis of 1,2,3-triazole-linked pyrrolobenzodiazepine conjugates employing 'click' chemistry: DNA-binding affinity and anticancer activity. Bioorg. Med. Chem. Lett. 2008, 18, 1468-1473. [CrossRef] [PubMed]

10. Singh, P.; Raj, R.; Kumar, V.; Mahajan, M.P.; Bedi, P.M.S.; Kaur, T.; Saxena, A.K. 1,2,3-Triazole tethered $\beta$-lactam-Chalcone bifunctional hybrids: Synthesis and anticancer evaluation. Eur. J. Med. Chem. 2012, 47, 594-600. [CrossRef] [PubMed]

11. Zhang, S.-Y.; Fu, D.-J.; Yue, X.-X.; Liu, Y.-C.; Song, J.; Sun, H.-H.; Liu, H.-M.; Zhang, Y.-B. Design, Synthesis and Structure-Activity Relationships of Novel Chalcone-1,2,3-triazole-azole Derivates as Antiproliferative Agents. Molecules 2016, 21, 653. [CrossRef] [PubMed]

12. Ye, W.; Yao, Q.; Yu, S.; Gong, P.; Qin, M. Synthesis and Antitumor Activity of Triazole-Containing Sorafenib Analogs. Molecules 2017, 22, 1759. [CrossRef] [PubMed]

13. Tiwari, V.K.; Mishra, B.B.; Mishra, K.B.; Mishra, N.; Singh, A.S.; Xi, C. Cu-Catalyzed Click Reaction in Carbohydrate Chemistry. Chem. Rev. 2016, 116, 3086-3240. [CrossRef] [PubMed]

14. Holstein, J.M.; Anhäuser, L.; Rentmeister, A. Modifying the 5'-Cap for Click Reactions of Eukaryotic mRNA and To Tune Translation Efficiency in Living Cells. Angew. Chem. Int. Ed. Engl. 2016, 55, 10899-10903. [CrossRef] [PubMed]

15. Fu, D.J.; Zhang, S.Y.; Liu, Y.C.; Zhang, L.; Liu, J.J.; Song, J.; Zhao, R.H.; Li, F.; Sun, H.H.; Liu, H.M. Design, synthesis and antiproliferative activity studies of novel dithiocarbamate-chalcone derivates. Bioorg. Med. Chem. Lett. 2016, 26, 3918-3922. [CrossRef] [PubMed]

16. Robertson, S.; Martinez, G.J.; Payet, C.A.; Barraclough, J.Y.; Celermajer, D.S.; Bursill, C.; Patel, S. Colchicine Therapy in Acute Coronary Syndrome Patients acts on Caspase-1 to Suppress NLRP3 Inflammasome Monocyte Activation. Clin. Sci. 2016, 130, 1237-1246. [CrossRef] [PubMed]

17. Ravelli, R.B.; Gigant, B.; Curmi, P.A.; Jourdain, I.; Lachkar, S.; Sobel, A.; Knossow, M. Insight into tubulin regulation from a complex with colchicine and a stathmin-like domain. Nature 2004, 428, 198-202. [CrossRef] [PubMed]

18. Arai, H.; Miyakawa, K.; Denda, T.; Mizukami, T.; Horie, Y.; Izawa, N.; Hirakawa, M.; Ogura, T.; Tsuda, T.; $\mathrm{Yu}, \mathrm{S}$. Early morphological change for predicting outcome in metastatic colorectal cancer after regorafenib. Oncotarget 2017, 8, 110530-110539. [CrossRef] [PubMed]

19. Gonçalves, B.M.F.; Salvador, J.A.R.; Marín, S.; Cascante, M. Synthesis and anticancer activity of novel fluorinated asiatic acid derivatives. Eur. J. Med. Chem. 2016, 114, 101-117. [CrossRef] [PubMed]

20. Czabotar, P.E.; Lessene, G.; Strasser, A.; Adams, J.M. Control of apoptosis by the BCL-2 protein family: Implications for physiology and therapy. Nat. Rev. Mol. Cell Biol. 2014, 15, 49-63. [CrossRef] [PubMed]

21. Inoueyamauchi, A.; Jeng, P.S.; Kim, K.; Chen, H.C.; Han, S.; Ganesan, Y.T.; Ishizawa, K.; Jebiwott, S.; Dong, Y.; Pietanza, M.C. Targeting the differential addiction to anti-apoptotic BCL-2 family for cancer therapy. Nat. Commn. 2017, 8, 16078. [CrossRef] [PubMed]

22. Kunciw, D.L.; Liechty, J.J.; Mitchell, M.O.; Wan, B.J.; Franzblau, S.G. Structural requirements for the antitubercular quaternized triflupromazine pharmacophore. Bioorg. Med. Chem. Lett. 2012, 22, 5679-5680. [CrossRef] [PubMed]

Sample Availability: Samples of the compounds are not available from the authors. 